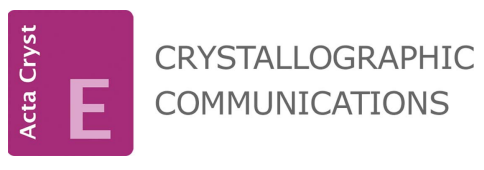

ISSN 2056-9890

Received 30 January 2018

Accepted 23 February 2018

Edited by W. T. A. Harrison, University of Aberdeen, Scotland

Keywords: cobalt(II); hybrid organic-inorganic materials; crystal structure; Hirshfeld surface; fingerprint plots.

CCDC reference: 1588020

Supporting information: this article has supporting information at journals.iucr.org/e

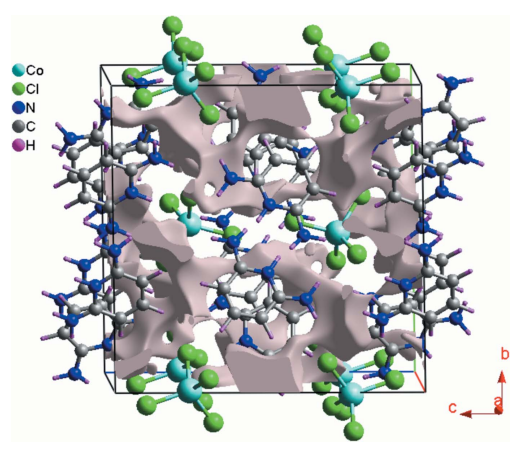

OPEN $\odot$ ACCESS

\section{Crystal structure and Hirshfeld surface analysis of bis(2,6-diaminopyridinium) tetrachlorido- cobaltate(II)}

\author{
Oumaima Ben Moussa, ${ }^{a}$ Hammouda Chebbi ${ }^{\mathrm{a}, \mathrm{b}}$ and Mohamed Faouzi Zid ${ }^{\mathrm{a} *}$ \\ a University of Tunis El Manar, Faculty of Sciences of Tunis, Laboratory of Materials, Crystal Chemistry and Applied \\ Thermodynamics, 2092 El Manar II, Tunis, Tunisia, and ${ }^{\mathbf{b}}$ University of Tunis, Preparatory Institute for Engineering Studies \\ of Tunis, Street Jawaher Lel Nehru, 1089 Montfleury, Tunis, Tunisia. *Correspondence e-mail: \\ medfaouzi.zid57@gmail.com
}

In the title molecular salt, $\left(\mathrm{C}_{5} \mathrm{H}_{8} \mathrm{~N}_{3}\right)_{2}\left[\mathrm{CoCl}_{4}\right]$, the cations are protonated at their pyridine $\mathrm{N}$ atoms and the anion is an almost regular tetrahedron. The crystal structure consists of alternating inorganic layers, built from tetrachloridocobaltate anions, and organic layers formed by protonated cations of 2,6diaminopyridinium. The crystal packing is governed by $\mathrm{C} / \mathrm{N}-\mathrm{H} \cdots \mathrm{Cl}$ hydrogenbonding interactions between the organic and the inorganic ions and $\mathrm{Cl} \cdots \mathrm{Cl}$ interactions. Moreover, the cations show a $\pi-\pi$ stacking interaction [intercentroid distance $=3.763(2) \AA]$. The prevalence of these interactions is illustrated by an analysis of the three-dimensional Hirshfeld surface and by twodimensional fingerprint plots.

\section{Chemical context}

One of the best studied groups of organic-inorganic hybrid materials are the cobalt(II) halide compounds because of their important properties such as fluorescence and magnetism (Decaroli et al., 2015; Kurmoo, 2009). The coordination sphere of $\mathrm{Co}^{\mathrm{II}}$ is variable, leading to different geometries including octahedral, tetrahedral, square pyramidal, trigonal bipyramidal and square planar (Kurmoo, 2009). Pyridine as an organic heterocyclic molecule has various biological activities (Sellin, 1981; Davidson et al., 1988). As part of our studies in this area, the title compound, $\left(\mathrm{C}_{5} \mathrm{H}_{8} \mathrm{~N}_{3}\right)_{2}\left[\mathrm{CoCl}_{4}\right]$ (I), has been investigated.

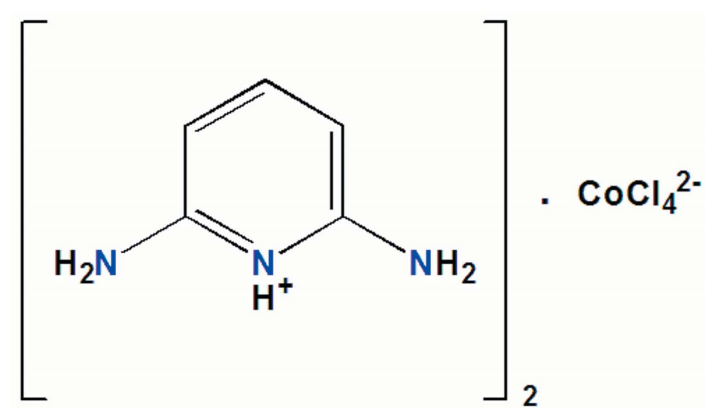

\section{Structural commentary}

The asymmetric unit of (I) is made up of one tetrachloridocobaltate, $\left[\mathrm{CoCl}_{4}\right]^{2-}$, anion and two protonated 2,6-diaminopyridinium, $\left(\mathrm{C}_{5} \mathrm{H}_{8} \mathrm{~N}_{3}\right)^{+}$, organic cations (Fig. 1). The geometry of the $\mathrm{CoCl}_{4}$ anion is characterized by a range of $\mathrm{Co}-\mathrm{Cl}$ bond 


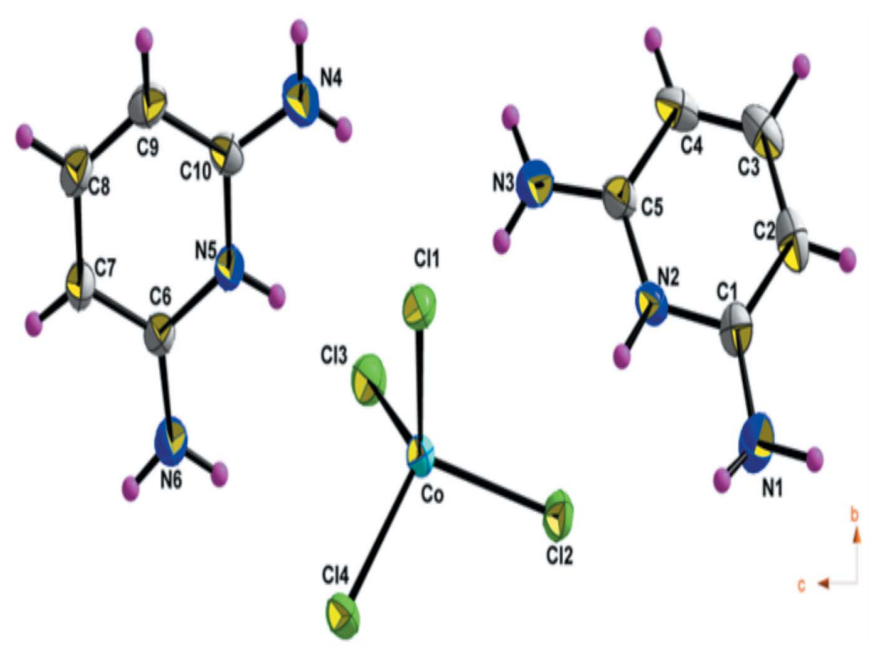

Figure 1

The asymmetric unit of (I), with displacement ellipsoids are drawn at the $30 \%$ probability level.

length from 2.2595 (14) to 2.2795 (13) $\AA$ and $\mathrm{Cl}-\mathrm{Co}-\mathrm{Cl}$ angles varying from $106.44(5)$ to $112.69(5)^{\circ}$, building a slightly distorted tetrahedron. These data are in agreement with those found in related compounds (Mghandef \& Boughzala, 2015). The calculated average values of the distortion indice as described by Baur (1974) corresponding to the different lengths and angles in the $\mathrm{CoCl}_{4}$ tetrahedra $[\Delta \mathrm{I}(\mathrm{Co}-\mathrm{Cl})=0.004$ and $\Delta \mathrm{I}(\mathrm{Cl}-\mathrm{Co}-\mathrm{Cl})=0.0019]$ show a slight distortion of the tetrahedra. The interanionic $\mathrm{Cl} \cdots \mathrm{Cl}$ contact distances between the nearest neighbor tetrahedra are 3.986 (2) $\AA$ along the $a$ axis and 3.889 (2) $\AA$ along the $c$ axis (Fig. 2), compared to a van der Waals contact distance of $3.50 \AA$. These contacts are sometimes associated with weak antiferromagnetic interactions (Shapiro et al., 2007), which decrease rapidly with increasing $\mathrm{Cl} \cdots \mathrm{Cl}$ separation.

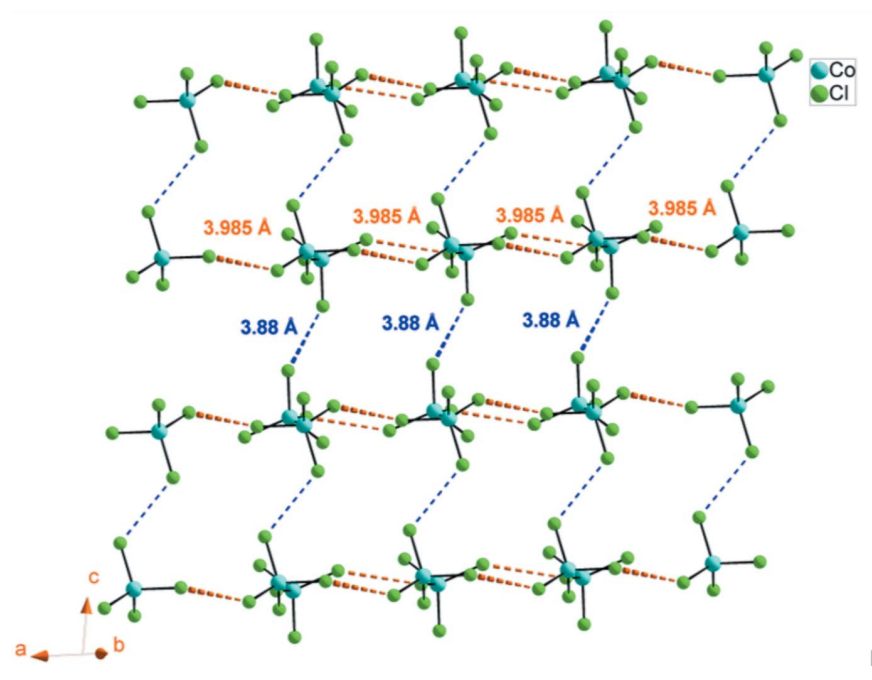

Figure 2

The interanionic $\mathrm{Cl} \cdots \mathrm{Cl}$ contact in the tetrachlorocobaltate anion of (I) along the $a$ and $c$ axis.
Table 1

Hydrogen-bond geometry $\left(\AA,^{\circ}\right)$.

\begin{tabular}{|c|c|c|c|c|}
\hline$D-\mathrm{H} \cdots A$ & $D-\mathrm{H}$ & $\mathrm{H} \cdots A$ & $D \cdots A$ & $D-\mathrm{H} \cdots A$ \\
\hline $\mathrm{N} 1-\mathrm{H} 2 N 1 \cdots \mathrm{Cl} 2$ & $0.84(5)$ & $2.69(5)$ & $3.432(4)$ & $147(4)$ \\
\hline $\mathrm{N} 4-\mathrm{H} 2 N 4 \cdots \mathrm{Cl} 2^{\mathrm{i}}$ & $0.84(5)$ & $2.65(5)$ & 3.465 (5) & $162(4)$ \\
\hline $\mathrm{N} 6-\mathrm{H} 1 N 6 \cdots \mathrm{Cl} 4$ & $0.85(5)$ & $2.58(5)$ & $3.406(4)$ & $165(4)$ \\
\hline $\mathrm{N} 2-\mathrm{H} 1 N 2 \cdots \mathrm{Cl} 2$ & $0.82(4)$ & $2.44(4)$ & $3.240(3)$ & $168(4)$ \\
\hline $\mathrm{N} 5-\mathrm{H} 1 N 5 \cdots \mathrm{Cl} 1$ & $0.82(5)$ & $2.43(5)$ & $3.191(3)$ & $156(4)$ \\
\hline $\mathrm{N} 3-\mathrm{H} 1 N 3 \cdots \mathrm{Cl} 4^{\mathrm{ii}}$ & $0.88(4)$ & $2.53(5)$ & $3.390(5)$ & $166(4)$ \\
\hline $\mathrm{N} 4-\mathrm{H} 1 N 4 \cdots \mathrm{Cl} 1$ & $0.79(4)$ & $2.79(4)$ & $3.481(5)$ & $147(4)$ \\
\hline $\mathrm{N} 6-\mathrm{H} 2 N 6 \cdots \mathrm{Cl} 3^{\mathrm{iii}}$ & $0.82(5)$ & $2.75(5)$ & $3.516(4)$ & $156(4)$ \\
\hline $\mathrm{N} 3-\mathrm{H} 2 N 3 \cdots \mathrm{Cl} 3$ & $0.83(6)$ & $2.61(6)$ & $3.420(5)$ & $166(6)$ \\
\hline $\mathrm{N} 1-\mathrm{H} 1 N 1 \cdots \mathrm{Cl} 1^{\mathrm{iv}}$ & $0.94(5)$ & $2.69(5)$ & $3.326(4)$ & $126(4)$ \\
\hline $\mathrm{C} 7-\mathrm{H} 7 \cdots \mathrm{Cl}^{\mathrm{iii}}$ & $0.91(4)$ & $2.89(4)$ & $3.669(4)$ & $145(3)$ \\
\hline
\end{tabular}

Pyridinium cations always possess an expanded angle of $\mathrm{C}-\mathrm{N}-\mathrm{C}$ in comparison with the parent pyridine (Ben Nasr et al., 2015). Thus, the observed angles in (I) of $\mathrm{C} 1-\mathrm{N} 2-\mathrm{C} 5$ and $\mathrm{C} 6-\mathrm{N} 5-\mathrm{C} 10$ are $124.2(3)$ and $124.1(3)^{\circ}$, respectively, are wider than that in neutral pyridine $\left(116.6^{\circ}\right)$, indicating that protonation takes place on the pyridine ring $\mathrm{N} 2$ and $\mathrm{N} 5$ atoms. Accordingly, within the cations, we note that the $\mathrm{N}-\mathrm{C}$ and $\mathrm{C}-\mathrm{C}$ distances range from 1.332 (5) to 1.393 (6) $\AA$, while the $\mathrm{C}-\mathrm{C}-\mathrm{C}, \mathrm{N}-\mathrm{C}-\mathrm{N}, \mathrm{C}-\mathrm{C}-\mathrm{N}$ and $\mathrm{N}-\mathrm{C}-\mathrm{C}$ angles vary from $116.00(4)$ to $126.50(4)^{\circ}$. The 2,6-diaminopyridinium units are essentially planar, with an r.m.s. deviation from the mean plane of 0.002 and $0.006 \AA$ for the N2 and N5 species, respectively.

\section{Supramolecular features}

Examination of the crystal structure of (I) reveals organic layers parallel to the $a b$ plane made of 2,6-diaminopyridinium cations alternating with inorganic layers formed by tetrachloridocobaltate anions (Fig. 3), which is similar to those of

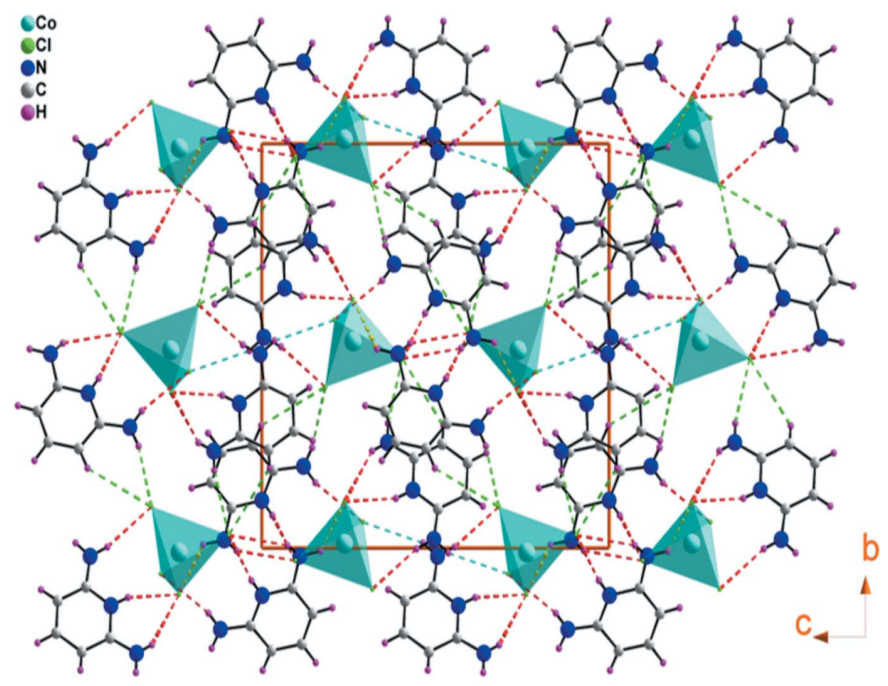

Figure 3

View of (I) towards the $b c$ plane. The dotted lines indicate hydrogen bonds. 


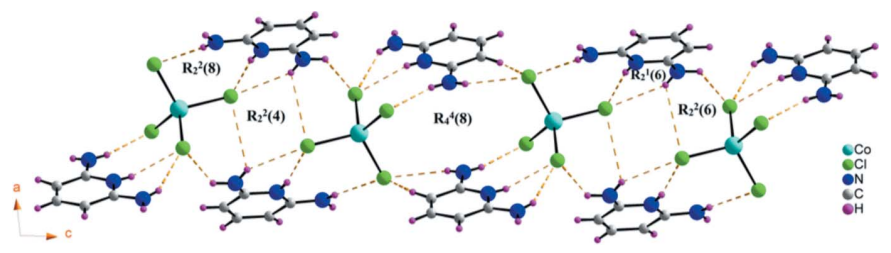

Figure 4

Projection of a part of the crystalline structure of the compound (I), showing the formation of the motifs $R_{2}^{2}(4), R_{2}^{2}(6), R_{2}^{1}(6), R_{4}^{4}(8)$ and $R_{2}^{2}(8)$.

related materials: $\left(\mathrm{C}_{5} \mathrm{H}_{6} \mathrm{Br}_{2} \mathrm{~N}_{3}\right)_{2}\left[M \mathrm{Br}_{4}\right](M=\mathrm{Cd}, \mathrm{Mn})(\mathrm{Al}-\mathrm{Far}$ et al., 2009) and $\left(\mathrm{C}_{5} \mathrm{H}_{7} \mathrm{~N}_{2}\right)_{2}\left[\mathrm{CoBr}_{4}\right]$ (Mhadhbi et al., 2016).

The construction of the three-dimensional architecture is consolidated by $\mathrm{N}-\mathrm{H} \cdots \mathrm{Cl}$ and $\mathrm{C}-\mathrm{H} \cdots \mathrm{Cl}$ hydrogen bonds (Table 1), generating $R_{2}^{2}(4), R_{2}^{2}(6), R_{2}^{1}(6), R_{4}^{4}(8)$ and $R_{2}^{2}(8)$ graph-set motifs (Fig. 4).

As can be seen from Fig. 5, the two nearest neighboring anti-parallel organic cations, which are not connected by hydrogen bonding, are stacked in a face-to-face mode. The centroid-centroid distance is $3.762(5) \AA$, slightly less than $3.8 \AA$, which is the maximum value accepted for $\pi-\pi$ interactions (Ben Hassen et al., 2017; Janiak, 2000).

\section{Hirshfeld surface analysis}

The Hirshfeld surface (Spackman \& Jayatilaka, 2009) and the associated two-dimensional fingerprint plots were performed with CrystalExplorer (Wolff et al., 2012). The Hirshfeld surface of the title compound mapped over $d_{\text {norm }}$ is illustrated in Fig. 6 . The red spots correspond to the $\mathrm{H} \cdots \mathrm{Cl}$ close contacts, which are due to the $\mathrm{N}-\mathrm{H} \cdots \mathrm{Cl}$ hydrogen bonds. Similarly, the presence of $\mathrm{H} \cdots \mathrm{Cl}$ contacts (due to $\mathrm{C}-\mathrm{H} \cdots \mathrm{Cl}$ hydrogen bonds) are indicated by a light-red color. The white areas correspond to the places where the distance separating neighboring atoms are close to the sum of the van der Waals radius of the considered atoms and indicate $\mathrm{H} \cdots \mathrm{H}$ interactions. The bluish areas illustrate areas where neighboring

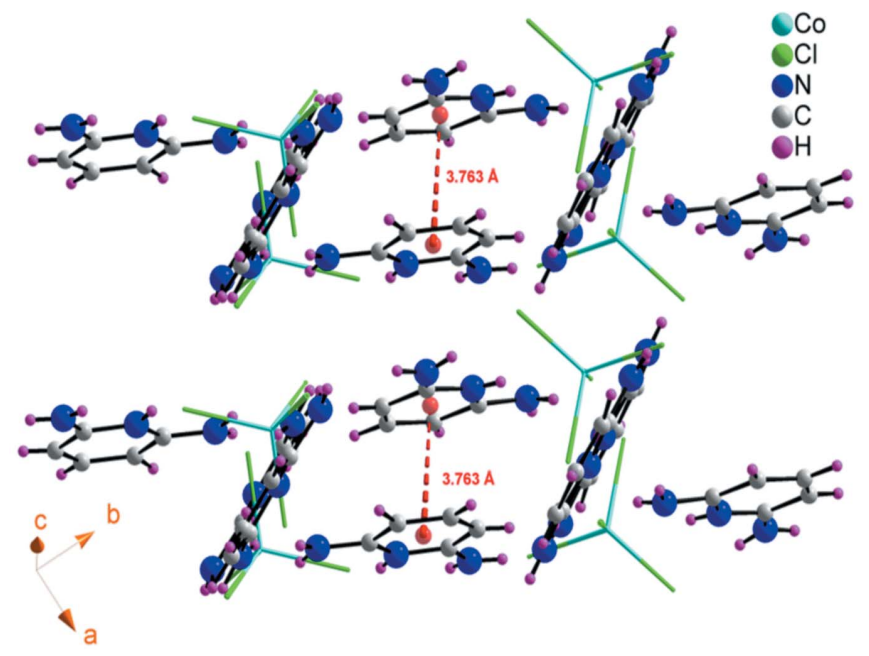

Figure 5

$\pi-\pi$ stacking interactions between the neighboring aromatic organic cations in (I). The inorganic anions are shown as sticks for clarity.

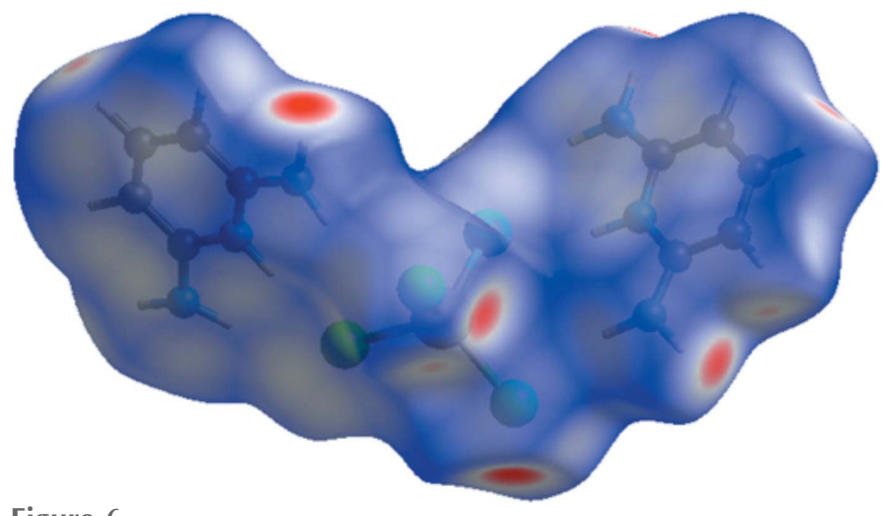

Figure 6

View of the Hirshfeld surface of (I) mapped over $d_{\text {norm }}$.

atoms are too far apart for there to be interactions between them. In the shape-index map (Fig. 7), the adjacent red and blue triangle-like patches show concave regions that indicate $\pi-\pi$ stacking interactions (Bitzer et al., 2017).

The fingerprint plots of (I) (Fig. 8a) (Parkin et al., 2007; Rohl et al., 2008), reveal that the main intermolecular interactions with the highest percentage contributions are: $\mathrm{H} \cdots \mathrm{Cl} /$ $\mathrm{Cl} \cdots \mathrm{H}(41.6 \%$, Fig. $8 b), \mathrm{H} \cdots \mathrm{H}(30.8 \%$, Fig. $8 c)$ and $\mathrm{C} \cdots \mathrm{H} /$ H..C (11.3\%, Fig. $8 d)$.

Fig. 9 shows the voids (Wolff et al., 2012) in the crystal structure of (I). These are based on the sum of spherical atomic electron densities at the appropriate nuclear positions (procrystal electron density). The crystal voids calculation (results under 0.002 a.u. isovalue) shows the void volume of title compound to be of the order of $172 \AA^{3}$ and surface area in the order of $648 \AA^{2}$. With the porosity, the calculated void volume of (I) is $10 \%$. There are no large cavities. We note that the electron-density isosurfaces are not completely closed around the components, but are open at those locations where interspecies approaches are found, e.g. $\mathrm{N}-\mathrm{H} \cdots \mathrm{Cl}$ and $\mathrm{C}-$ $\mathrm{H} \cdots \mathrm{Cl}$.

\section{Synthesis and crystallization}

2,6-diaminopyridine and $\mathrm{CoCl}_{2} \cdot 6 \mathrm{H}_{2} \mathrm{O}$ (molar ratio 1:1) were dissolved in $10 \mathrm{ml}$ of methanol; $3 \mathrm{ml}$ of hydrochloric acid

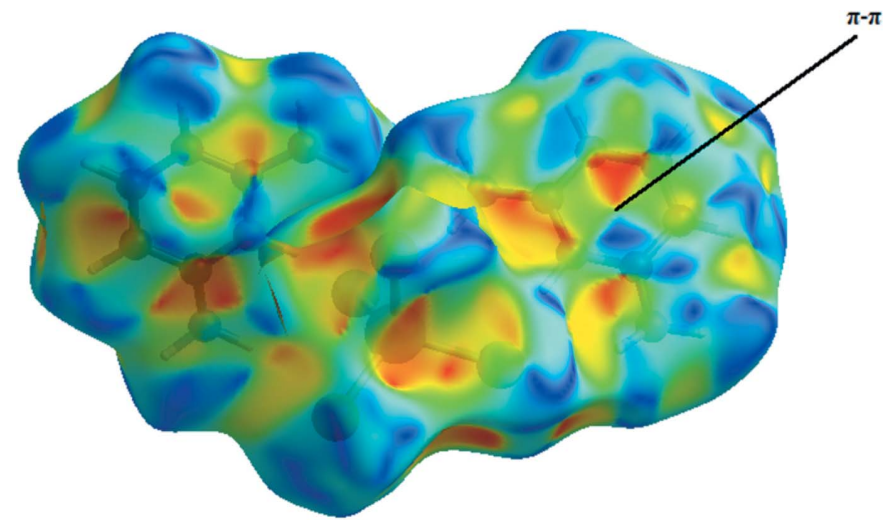

Figure 7

Hirshfeld surface mapped over shape-index, highlighting the regions involved in $\pi-\pi$ stacking interactions. 
(a)

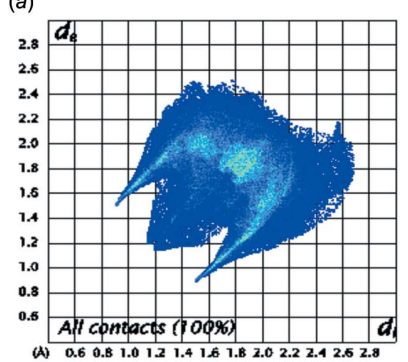

(c)

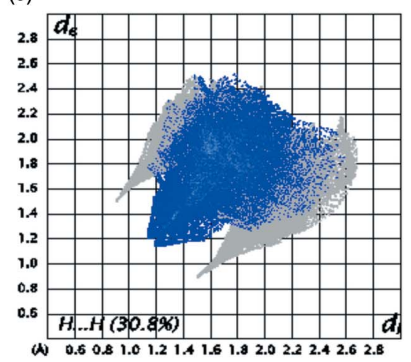

(b)

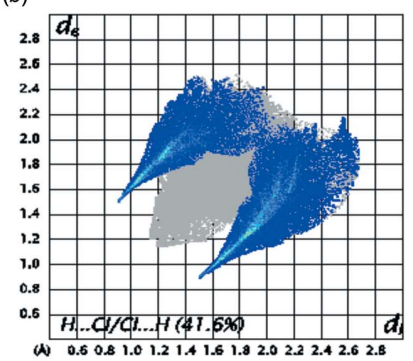

(d)

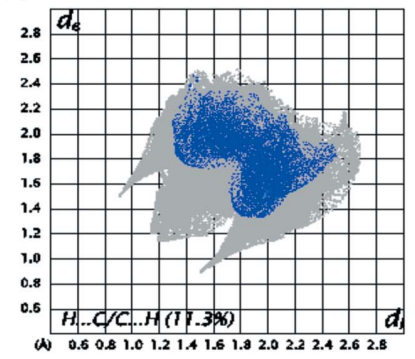

Figure 8

The two-dimensional fingerprint plots of (I), (a) showing all interactions and delineated into $\mathrm{H} \cdots \mathrm{Cl} / \mathrm{Cl} \cdots \mathrm{H}(b), \mathrm{H} \cdots \mathrm{H}(c)$ and $\mathrm{C} \cdots \mathrm{H} / \mathrm{H} \cdots \mathrm{C}(d)$ interactions.

(37\%) was added dropwise to the mixture and the resulting blue solution was put aside for crystallization at room temperature. After two weeks, blue crystals of (I) were recovered.

\section{Refinement}

Crystal data, data collection and structure refinement details are summarized in Table 2. All hydrogen atoms were found in a difference-Fourier map and refined isotropically.

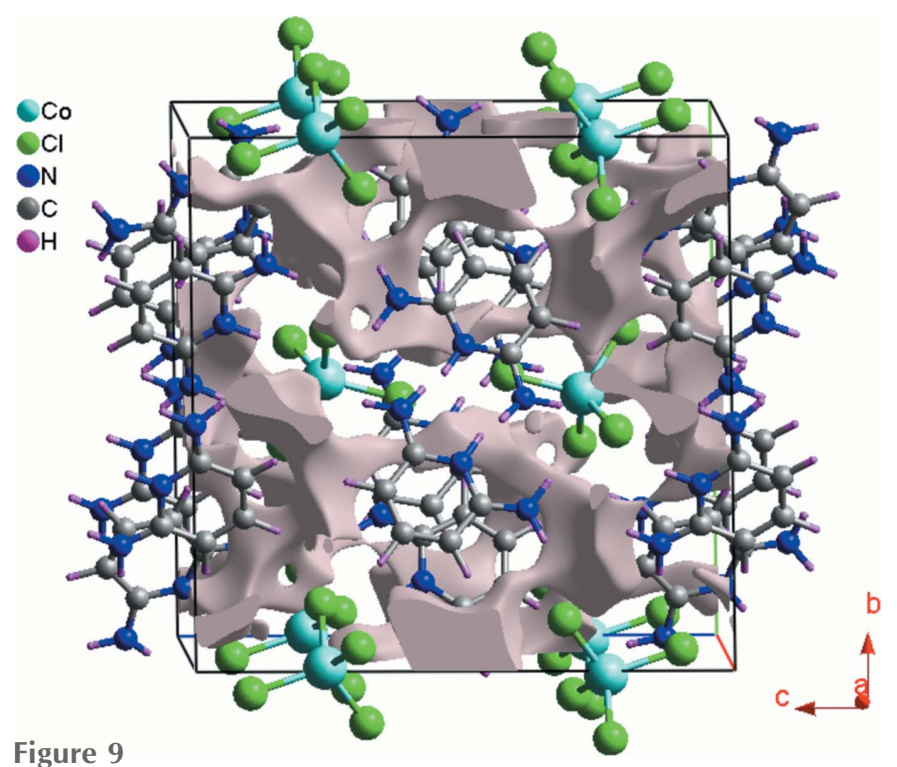

Void plot for (I).

Table 2

Experimental details.

Crystal data

Chemical formula

$M_{\mathrm{r}}$

Crystal system, space group

Temperature (K)

$a, b, c(\AA)$

$\beta\left({ }^{\circ}\right)$

$V\left(\AA^{3}\right)$

$Z$

Radiation type

$\mu\left(\mathrm{mm}^{-1}\right)$

Crystal size (mm)

$\left(\mathrm{C}_{5} \mathrm{H}_{8} \mathrm{~N}_{3}\right)_{2}\left[\mathrm{CoCl}_{4}\right]$

421.02

Monoclinic, $P 2_{1} / n$

293

7.390 (4), 15.373 (4), 15.387 (4)

98.203 (4)

$1730.1(11)$

Mo $K \alpha$

1.61

$0.4 \times 0.3 \times 0.1$

Data collection

Diffractometer

Absorption correction

$T_{\min }, T_{\max }$

No. of measured, independent and observed $[I>2 \sigma(I)]$ reflections

$R_{\text {int }}$

$(\sin \theta / \lambda)_{\max }\left(\AA^{-1}\right)$

Enraf-Nonius CAD-4

$\psi$ scan (North et al., 1968)

$0.777,0.998$

4367, 3770, 2396

0.038

0.638

Refinement

$R\left[F^{2}>2 \sigma\left(F^{2}\right)\right], w R\left(F^{2}\right), S$

No. of reflections

No. of parameters

$\mathrm{H}$-atom treatment

$\Delta \rho_{\max }, \Delta \rho_{\min }\left(\mathrm{e}^{-3}\right)$

$0.044,0.110,1.01$

3770

255

All $\mathrm{H}$-atom parameters refined $0.37,-0.42$

Computer programs: CAD-4 EXPRESS (Duisenberg, 1992; Macíček \& Yordanov, 1992), XCAD4 (Harms \& Wocadlo, 1995), SHELXS97 (Sheldrick, 2008), SHELXL2014 (Sheldrick, 2015), DIAMOND (Brandenburg, 2006), WinGX (Farrugia, 2012) and publCIF (Westrip, 2010).

\section{Funding information}

The authors acknowledge financial support from the Ministry of Higher Education and Scientific Research of Tunisia.

\section{References}

Al-Far, R. H., Haddad, S. F. \& Ali, B. F. (2009). Acta Cryst. C65, m321-m324.

Baur, W. H. (1974). Acta Cryst. B30, 1195-1215.

Ben Hassen, C., Dammak, T., Chniba-Boudjada, N., Mhiri, T. \& Boujelbene, M. (2017). J. Mol. Struct. 1127, 43-52.

Ben Nasr, M., Lefebvre, F. \& Ben Nasr, C. (2015). Am. J. Anal. Chem. 6, 446-456.

Bitzer, S. R., Visentin, C. L., Hörner, M., Nascimento, M. A. C. \& Filgueiras, C. A. L. (2017). J. Mol. Struct. 1130, 165-173.

Brandenburg, K. (2006). DIAMOND. Crystal Impact GbR, Bonn, Germany.

Davidson, M., Zemishlany, Z., Mohs, R. C., Horvath, T. B., Powchik, P., Blass, J. P. \& Davis, K. L. (1988). Biol. Psychiatry, 23, 485490.

Decaroli, C., Arevalo-Lopez, A. M., Woodall, C. H., Rodriguez, E. E., Attfield, J. P., Parker, S. F. \& Stock, C. (2015). Acta Cryst. B71, 2024.

Duisenberg, A. J. M. (1992). J. Appl. Cryst. 25, 92-96.

Farrugia, L. J. (2012). J. Appl. Cryst. 45, 849-854.

Harms, K. \& Wocadlo, S. (1995). XCAD4. University of Marburg, Germany.

Janiak, C. (2000). J. Chem. Soc. Dalton Trans. pp. 3885-3896.

Kurmoo, M. (2009). Chem. Soc. Rev. 38, 1353-1379.

Macíček, J. \& Yordanov, A. (1992). J. Appl. Cryst. 25, 73-80.

Mghandef, M. \& Boughzala, H. (2015). Acta Cryst. E71, 555557. 
Mhadhbi, N., Saïd, S., Elleuch, S. \& Naili, H. (2016). J. Mol. Struct. 1108, 223-234.

North, A. C. T., Phillips, D. C. \& Mathews, F. S. (1968). Acta Cryst. A24, 351-359.

Parkin, A., Barr, G., Dong, W., Gilmore, C. J., Jayatilaka, D., McKinnon, J. J., Spackman, M. A. \& Wilson, C. C. (2007). CrystEngComm, 9, 648-652.

Rohl, A. L., Moret, M., Kaminsky, W., Claborn, K., McKinnon, J. J. \& Kahr, B. (2008). Cryst. Growth Des. 8, 4517-4525.

Sellin, L. C. (1981). Med. Biol. 59, 11-20.
Shapiro, A., Landee, C. P., Turnbull, M. M., Jornet, J., Deumal, M., Novoa, J. J., Robb, M. A. \& Lewis, W. (2007). J. Am. Chem. Soc. 129, 952-959.

Sheldrick, G. M. (2008). Acta Cryst. A64, 112-122.

Sheldrick, G. M. (2015). Acta Cryst. C71, 3-8.

Spackman, M. A. \& Jayatilaka, D. (2009). CrystEngComm, 11, 19-32.

Westrip, S. P. (2010). J. Appl. Cryst. 43, 920-925.

Wolff, S. K., Grimwood, D. J., McKinnon, J. J., Turner, M. J., Jayatilaka, D. \& Spackman, M. A. (2012). Crystal Explorer. University of Western Australia. 


\section{supporting information}

Acta Cryst. (2018). E74, 436-440 [https://doi.org/10.1107/S2056989018003171]

\section{Crystal structure and Hirshfeld surface analysis of bis(2,6-diaminopyridinium) tetrachloridocobaltate(II)}

\section{Oumaima Ben Moussa, Hammouda Chebbi and Mohamed Faouzi Zid}

Computing details

Data collection: CAD-4 EXPRESS (Duisenberg, 1992; Macíček \& Yordanov, 1992); cell refinement: CAD-4 EXPRESS

(Duisenberg, 1992; Macíček \& Yordanov, 1992); data reduction: XCAD4 (Harms \& Wocadlo, 1995); program(s) used to solve structure: SHELXS97 (Sheldrick, 2008); program(s) used to refine structure: SHELXL2014 (Sheldrick, 2015); molecular graphics: DIAMOND (Brandenburg, 2006); software used to prepare material for publication: WinGX (Farrugia, 2012) and publCIF (Westrip, 2010).

Bis(2,6-diaminopyridinium) tetrachloridocobaltate(II)

Crystal data

$\left(\mathrm{C}_{5} \mathrm{H}_{8} \mathrm{~N}_{3}\right)_{2}\left[\mathrm{CoCl}_{4}\right]$

$M_{r}=421.02$

Monoclinic, $P 2_{1} / n$

$a=7.390$ (4) $\AA$

$b=15.373(4) \AA$

$c=15.387(4) \AA$

$\beta=98.203(4)^{\circ}$

$V=1730.1(11) \AA^{3}$

$Z=4$

$F(000)=852$

$$
\begin{gathered}
D_{\mathrm{x}}=1.616 \mathrm{Mg} \mathrm{m}^{-3} \\
D_{\mathrm{m}}=1.616 \mathrm{Mg} \mathrm{m}^{-3} \\
D_{\mathrm{m}} \text { measured by } ?
\end{gathered}
$$

Mo $K \alpha$ radiation, $\lambda=0.71073 \AA$

Cell parameters from 25 reflections

$\theta=10.1-14.9^{\circ}$

$\mu=1.61 \mathrm{~mm}^{-1}$

$T=293 \mathrm{~K}$

Parallelepiped, blue

$0.4 \times 0.3 \times 0.1 \mathrm{~mm}$

\section{Data collection}

Enraf-Nonius CAD-4

diffractometer

Radiation source: fine-focus sealed tube

Graphite monochromator

$\omega / 2 \theta$ scans

Absorption correction: $\psi$ scan

(North et al., 1968)

$T_{\min }=0.777, T_{\max }=0.998$

4367 measured reflections

\section{Refinement}

Refinement on $F^{2}$

Least-squares matrix: full

$R\left[F^{2}>2 \sigma\left(F^{2}\right)\right]=0.044$

$w R\left(F^{2}\right)=0.110$

$S=1.01$

3770 reflections

$\theta_{\text {max }}=27.0^{\circ}, \theta_{\min }=2.7^{\circ}$
3770 independent reflections

2396 reflections with $I>2 \sigma(I)$

$R_{\text {int }}=0.038$

$h=-9 \rightarrow 1$

$k=-1 \rightarrow 19$

$l=-19 \rightarrow 19$

2 standard reflections every 120 reflections intensity decay: $1 \%$

\section{5 parameters}

0 restraints

Hydrogen site location: difference Fourier map

All $\mathrm{H}$-atom parameters refined

$w=1 /\left[\sigma^{2}\left(F_{0}^{2}\right)+(0.0522 P)^{2}\right]$

where $P=\left(F_{\mathrm{o}}^{2}+2 F_{\mathrm{c}}^{2}\right) / 3$ 
$(\Delta / \sigma)_{\max }=0.001$

$\Delta \rho_{\max }=0.37 \mathrm{e} \AA^{-3}$

$\Delta \rho_{\min }=-0.42$ e $\AA^{-3}$
Extinction correction: SHELXL2014 (Sheldrick, 2015),

$\mathrm{Fc}^{*}=\mathrm{kFc}\left[1+0.001 \mathrm{xFc}^{2} \lambda^{3} / \sin (2 \theta)\right]^{-1 / 4}$

Extinction coefficient: $0.0049(8)$

\section{Special details}

Geometry. All esds (except the esd in the dihedral angle between two 1.s. planes) are estimated using the full covariance matrix. The cell esds are taken into account individually in the estimation of esds in distances, angles and torsion angles; correlations between esds in cell parameters are only used when they are defined by crystal symmetry. An approximate (isotropic) treatment of cell esds is used for estimating esds involving l.s. planes.

Fractional atomic coordinates and isotropic or equivalent isotropic displacement parameters $\left(\AA^{2}\right)$

\begin{tabular}{|c|c|c|c|c|}
\hline & $x$ & $y$ & $z$ & $U_{\text {iso }} * / U_{\text {eq }}$ \\
\hline Col & $0.44824(7)$ & $0.51081(3)$ & $0.73764(3)$ & $0.03878(16)$ \\
\hline $\mathrm{C} 11$ & $0.66787(14)$ & $0.61509(6)$ & $0.73884(6)$ & $0.0497(2)$ \\
\hline $\mathrm{Cl} 2$ & $0.36703(14)$ & $0.46854(6)$ & $0.59572(6)$ & $0.0500(3)$ \\
\hline $\mathrm{Cl3}$ & $0.20113(14)$ & $0.56398(7)$ & $0.79102(6)$ & $0.0547(3)$ \\
\hline $\mathrm{Cl} 4$ & $0.58007(17)$ & $0.39816(6)$ & $0.81784(7)$ & $0.0627(3)$ \\
\hline N2 & $0.1002(5)$ & $0.6175(2)$ & $0.4994(2)$ & $0.0451(7)$ \\
\hline N5 & $0.8682(4)$ & $0.6428(2)$ & $0.9347(2)$ & $0.0425(7)$ \\
\hline N4 & $0.9624(6)$ & $0.7606(3)$ & $0.8611(3)$ & $0.0623(10)$ \\
\hline N1 & $0.1431(6)$ & $0.5199(3)$ & $0.3912(3)$ & $0.0646(10)$ \\
\hline N6 & $0.7523(6)$ & $0.5209(2)$ & $0.9947(3)$ & $0.0678(12)$ \\
\hline N3 & $0.0650(7)$ & $0.7017(3)$ & $0.6196(3)$ & $0.0759(13)$ \\
\hline C6 & $0.8422(5)$ & $0.5964(2)$ & $1.0067(2)$ & $0.0444(8)$ \\
\hline $\mathrm{C} 10$ & $0.9484(5)$ & $0.7226(2)$ & $0.9377(2)$ & $0.0434(8)$ \\
\hline $\mathrm{C} 1$ & $0.0856(5)$ & $0.5995(3)$ & $0.4122(2)$ & $0.0483(9)$ \\
\hline $\mathrm{C} 7$ & $0.9051(6)$ & 0.6309 & $1.0881(2)$ & $0.0571(11)$ \\
\hline $\mathrm{C} 5$ & $0.0425(6)$ & $0.6919(2)$ & 0.5325 & $0.0489(9)$ \\
\hline $\mathrm{C} 9$ & $1.0121(6)$ & $0.7571(3)$ & $1.0189(3)$ & $0.0532(10)$ \\
\hline $\mathrm{C} 8$ & $0.9889(6)$ & $0.7105(3)$ & $1.0922(3)$ & $0.0581(11)$ \\
\hline $\mathrm{C} 2$ & $0.0069(6)$ & $0.6621(4)$ & $0.3543(3)$ & $0.0630(12)$ \\
\hline $\mathrm{C} 3$ & $-0.0514(6)$ & 0.7378 & $0.3851(3)$ & $0.0651(13)$ \\
\hline $\mathrm{C} 4$ & $-0.0359(6)$ & $0.7541(3)$ & $0.4729(4)$ & $0.0606(12)$ \\
\hline H7 & $0.874(6)$ & $0.603(3)$ & $1.136(3)$ & $0.066(13)^{*}$ \\
\hline $\mathrm{H} 2$ & $0.000(6)$ & $0.648(3)$ & $0.298(3)$ & $0.074(14)^{*}$ \\
\hline H4 & $-0.066(5)$ & $0.803(3)$ & $0.498(2)$ & $0.047(11)^{*}$ \\
\hline H9 & $1.071(6)$ & $0.802(3)$ & $1.022(3)$ & $0.075(15)^{*}$ \\
\hline H8 & $1.035(6)$ & $0.735(3)$ & $1.146(3)$ & $0.062(12)^{*}$ \\
\hline H3 & $-0.108(7)$ & $0.784(3)$ & $0.345(3)$ & $0.094(16)^{*}$ \\
\hline $\mathrm{H} 2 \mathrm{~N} 1$ & $0.222(6)$ & $0.493(3)$ & $0.427(3)$ & $0.061(14)^{*}$ \\
\hline $\mathrm{H} 2 \mathrm{~N} 4$ & $1.020(6)$ & $0.808(3)$ & $0.863(3)$ & $0.067(14)^{*}$ \\
\hline H1N6 & $0.720(7)$ & $0.498(3)$ & $0.945(3)$ & $0.074(15)^{*}$ \\
\hline H1N2 & $0.157(6)$ & $0.581(3)$ & $0.530(3)$ & $0.058(13)^{*}$ \\
\hline H1N5 & $0.839(6)$ & $0.623(3)$ & $0.886(3)$ & $0.079(16)^{*}$ \\
\hline $\mathrm{H} 1 \mathrm{~N} 3$ & $0.026(6)$ & $0.748(3)$ & $0.645(3)$ & $0.062(13)^{*}$ \\
\hline H1N4 & $0.913(6)$ & $0.740(3)$ & 0.817 (3) & $0.047(13)^{*}$ \\
\hline $\mathrm{H} 2 \mathrm{~N} 6$ & $0.747(7)$ & $0.488(3)$ & $1.036(3)$ & $0.082(17)^{*}$ \\
\hline
\end{tabular}




\begin{tabular}{lllll}
$\mathrm{H} 2 \mathrm{~N} 3$ & $0.095(9)$ & $0.661(4)$ & $0.654(4)$ & $0.12(2)^{*}$ \\
$\mathrm{H} 1 \mathrm{~N} 1$ & $0.139(7)$ & $0.508(3)$ & $0.331(3)$ & $0.089(17)^{*}$ \\
\hline
\end{tabular}

Atomic displacement parameters $\left(\hat{A}^{2}\right)$

\begin{tabular}{lllllll}
\hline & $U^{11}$ & $U^{22}$ & $U^{33}$ & $U^{12}$ & $U^{13}$ & $U^{23}$ \\
\hline Co1 & $0.0442(3)$ & $0.0358(3)$ & $0.0350(2)$ & $0.0012(2)$ & $0.00079(19)$ & $0.00112(19)$ \\
C11 & $0.0558(6)$ & $0.0446(5)$ & $0.0472(5)$ & $-0.0080(4)$ & $0.0022(4)$ & $-0.0022(4)$ \\
C12 & $0.0553(6)$ & $0.0531(5)$ & $0.0382(5)$ & $0.0072(4)$ & $-0.0049(4)$ & $-0.0042(4)$ \\
C13 & $0.0573(6)$ & $0.0547(6)$ & $0.0547(5)$ & $0.0058(5)$ & $0.0170(5)$ & $0.0013(5)$ \\
C14 & $0.0892(8)$ & $0.0407(5)$ & $0.0511(6)$ & $0.0053(5)$ & $-0.0141(5)$ & $0.0073(4)$ \\
N2 & $0.0487(19)$ & $0.0386(16)$ & $0.0468(17)$ & $0.0056(15)$ & $0.0027(15)$ & $0.0068(14)$ \\
N5 & $0.0458(18)$ & $0.0446(17)$ & $0.0360(16)$ & $-0.0034(14)$ & $0.0023(13)$ & $-0.0017(14)$ \\
N4 & $0.068(3)$ & $0.059(2)$ & $0.059(2)$ & $-0.015(2)$ & $0.006(2)$ & $0.010(2)$ \\
N1 & $0.061(2)$ & $0.077(3)$ & $0.054(2)$ & $0.011(2)$ & $0.0023(19)$ & $-0.011(2)$ \\
N6 & $0.103(3)$ & $0.053(2)$ & $0.045(2)$ & $-0.028(2)$ & $0.001(2)$ & $-0.0006(18)$ \\
N3 & $0.122(4)$ & $0.047(2)$ & $0.063(3)$ & $0.011(2)$ & $0.030(3)$ & $-0.001(2)$ \\
C6 & $0.051(2)$ & $0.0416(19)$ & $0.0397(19)$ & $-0.0056(17)$ & $0.0037(16)$ & $-0.0002(15)$ \\
C10 & $0.040(2)$ & $0.041(2)$ & $0.049(2)$ & $0.0030(16)$ & $0.0073(16)$ & $0.0048(16)$ \\
C1 & $0.039(2)$ & $0.057(2)$ & $0.048(2)$ & $-0.0024(18)$ & $0.0032(16)$ & $0.0011(18)$ \\
C7 & $0.078(3)$ & $0.057(3)$ & $0.035(2)$ & $-0.015(2)$ & $0.004(2)$ & $-0.0021(18)$ \\
C5 & $0.054(2)$ & $0.041(2)$ & $0.053(2)$ & $0.0032(18)$ & $0.0151(18)$ & $0.0070(18)$ \\
C9 & $0.050(2)$ & $0.046(2)$ & $0.062(3)$ & $-0.013(2)$ & $0.002(2)$ & $-0.0082(19)$ \\
C8 & $0.070(3)$ & $0.058(3)$ & $0.044(2)$ & $-0.014(2)$ & $-0.002(2)$ & $-0.0083(19)$ \\
C2 & $0.054(3)$ & $0.085(3)$ & $0.047(2)$ & $-0.006(2)$ & $0.000(2)$ & $0.016(2)$ \\
C3 & $0.060(3)$ & $0.061(3)$ & $0.073(3)$ & $0.001(2)$ & $0.005(2)$ & $0.029(2)$ \\
C4 & $0.059(3)$ & $0.040(2)$ & $0.085(3)$ & $0.009(2)$ & $0.019(2)$ & $0.015(2)$ \\
& & & & & &
\end{tabular}

Geometric parameters $\left(\AA,^{\circ}\right)$

\begin{tabular}{llll}
\hline $\mathrm{Co} 1-\mathrm{Cl} 3$ & $2.2595(14)$ & $\mathrm{N} 6-\mathrm{H} 2 \mathrm{~N} 6$ & $0.82(5)$ \\
$\mathrm{Co} 1-\mathrm{Cl} 4$ & $2.2645(11)$ & $\mathrm{N} 3-\mathrm{C} 5$ & $1.335(6)$ \\
$\mathrm{Co} 1-\mathrm{Cl} 2$ & $2.2754(11)$ & $\mathrm{N} 3-\mathrm{H} 1 \mathrm{~N} 3$ & $0.88(4)$ \\
$\mathrm{Co} 1-\mathrm{Cl1}$ & $2.2795(13)$ & $\mathrm{N} 3-\mathrm{H} 2 \mathrm{~N} 3$ & $0.83(6)$ \\
$\mathrm{N} 2-\mathrm{C} 5$ & $1.346(5)$ & $\mathrm{C} 6-\mathrm{C} 7$ & $1.379(5)$ \\
$\mathrm{N} 2-\mathrm{C} 1$ & $1.359(5)$ & $\mathrm{C} 10-\mathrm{C} 9$ & $1.378(5)$ \\
$\mathrm{N} 2-\mathrm{H} 1 \mathrm{~N} 2$ & $0.82(4)$ & $\mathrm{C} 1-\mathrm{C} 2$ & $1.382(6)$ \\
$\mathrm{N} 5-\mathrm{C} 6$ & $1.355(4)$ & $\mathrm{C} 7-\mathrm{C} 8$ & $1.369(6)$ \\
$\mathrm{N} 5-\mathrm{C} 10$ & $1.360(5)$ & $\mathrm{C} 7-\mathrm{H} 7$ & $0.91(4)$ \\
$\mathrm{N} 5-\mathrm{H} 1 \mathrm{~N} 5$ & $0.82(5)$ & $\mathrm{C} 5-\mathrm{C} 4$ & $1.393(6)$ \\
$\mathrm{N} 4-\mathrm{C} 10$ & $1.332(5)$ & $\mathrm{C} 9-\mathrm{C} 8$ & $1.367(6)$ \\
$\mathrm{N} 4-\mathrm{H} 2 \mathrm{~N} 4$ & $0.84(5)$ & $\mathrm{C} 9-\mathrm{H} 9$ & $0.82(5)$ \\
$\mathrm{N} 4-\mathrm{H} 1 \mathrm{~N} 4$ & $0.79(4)$ & $\mathrm{C} 8-\mathrm{H} 8$ & $0.93(4)$ \\
$\mathrm{N} 1-\mathrm{C} 1$ & $1.349(5)$ & $\mathrm{C} 2-\mathrm{C} 3$ & $1.350(7)$ \\
$\mathrm{N} 1-\mathrm{H} 2 \mathrm{~N} 1$ & $0.84(5)$ & $\mathrm{C} 2-\mathrm{H} 2$ & $0.89(4)$ \\
$\mathrm{N} 1-\mathrm{H} 1 \mathrm{~N} 1$ & $0.94(5)$ & $\mathrm{C} 3-\mathrm{C} 4$ & $1.362(7)$ \\
$\mathrm{N} 6-\mathrm{C} 6$ & $1.337(5)$ & $\mathrm{C} 3-\mathrm{H} 3$ & $0.99(5)$ \\
$\mathrm{N} 6-\mathrm{H} 1 \mathrm{~N} 6$ & $0.85(5)$ & $\mathrm{C} 4-\mathrm{H} 4$ & $0.89(4)$
\end{tabular}




\begin{tabular}{|c|c|c|c|}
\hline $\mathrm{Cl} 3-\mathrm{Co} 1-\mathrm{Cl} 4$ & $112.69(5)$ & $\mathrm{N} 4-\mathrm{C} 10-\mathrm{N} 5$ & $117.1(4)$ \\
\hline $\mathrm{Cl} 3-\mathrm{Co} 1-\mathrm{Cl} 2$ & $109.64(5)$ & $\mathrm{N} 4-\mathrm{C} 10-\mathrm{C} 9$ & $125.0(4)$ \\
\hline $\mathrm{Cl} 4-\mathrm{Co} 1-\mathrm{Cl} 2$ & $109.80(4)$ & $\mathrm{N} 5-\mathrm{C} 10-\mathrm{C} 9$ & $117.9(3)$ \\
\hline $\mathrm{Cl} 3-\mathrm{Co} 1-\mathrm{Cl} 1$ & $110.73(5)$ & $\mathrm{N} 1-\mathrm{C} 1-\mathrm{N} 2$ & $116.0(4)$ \\
\hline $\mathrm{Cl} 4-\mathrm{Co} 1-\mathrm{Cl} 1$ & $106.44(5)$ & $\mathrm{N} 1-\mathrm{C} 1-\mathrm{C} 2$ & $126.5(4)$ \\
\hline $\mathrm{Cl} 2-\mathrm{Co} 1-\mathrm{Cl} 1$ & $107.37(4)$ & $\mathrm{N} 2-\mathrm{C} 1-\mathrm{C} 2$ & $117.4(4)$ \\
\hline $\mathrm{C} 5-\mathrm{N} 2-\mathrm{C} 1$ & $124.2(3)$ & $\mathrm{C} 8-\mathrm{C} 7-\mathrm{C} 6$ & $118.5(4)$ \\
\hline $\mathrm{C} 5-\mathrm{N} 2-\mathrm{H} 1 \mathrm{~N} 2$ & $123(3)$ & $\mathrm{C} 8-\mathrm{C} 7-\mathrm{H} 7$ & $123(3)$ \\
\hline $\mathrm{C} 1-\mathrm{N} 2-\mathrm{H} 1 \mathrm{~N} 2$ & $113(3)$ & $\mathrm{C} 6-\mathrm{C} 7-\mathrm{H} 7$ & $118(3)$ \\
\hline $\mathrm{C} 6-\mathrm{N} 5-\mathrm{C} 10$ & $124.1(3)$ & $\mathrm{N} 3-\mathrm{C} 5-\mathrm{N} 2$ & $118.3(4)$ \\
\hline $\mathrm{C} 6-\mathrm{N} 5-\mathrm{H} 1 \mathrm{~N} 5$ & $120(3)$ & $\mathrm{N} 3-\mathrm{C} 5-\mathrm{C} 4$ & $124.4(4)$ \\
\hline $\mathrm{C} 10-\mathrm{N} 5-\mathrm{H} 1 \mathrm{~N} 5$ & $116(3)$ & $\mathrm{N} 2-\mathrm{C} 5-\mathrm{C} 4$ & $117.3(4)$ \\
\hline $\mathrm{C} 10-\mathrm{N} 4-\mathrm{H} 2 \mathrm{~N} 4$ & $117(3)$ & $\mathrm{C} 8-\mathrm{C} 9-\mathrm{C} 10$ & $118.7(4)$ \\
\hline $\mathrm{C} 10-\mathrm{N} 4-\mathrm{H} 1 \mathrm{~N} 4$ & $120(3)$ & $\mathrm{C} 8-\mathrm{C} 9-\mathrm{H} 9$ & $121(3)$ \\
\hline $\mathrm{H} 2 \mathrm{~N} 4-\mathrm{N} 4-\mathrm{H} 1 \mathrm{~N} 4$ & $123(4)$ & $\mathrm{C} 10-\mathrm{C} 9-\mathrm{H} 9$ & $120(3)$ \\
\hline $\mathrm{C} 1-\mathrm{N} 1-\mathrm{H} 2 \mathrm{~N} 1$ & $119(3)$ & $\mathrm{C} 9-\mathrm{C} 8-\mathrm{C} 7$ & $122.7(4)$ \\
\hline $\mathrm{C} 1-\mathrm{N} 1-\mathrm{H} 1 \mathrm{~N} 1$ & $117(3)$ & $\mathrm{C} 9-\mathrm{C} 8-\mathrm{H} 8$ & $117(3)$ \\
\hline $\mathrm{H} 2 \mathrm{~N} 1-\mathrm{N} 1-\mathrm{H} 1 \mathrm{~N} 1$ & $118(4)$ & $\mathrm{C} 7-\mathrm{C} 8-\mathrm{H} 8$ & $121(3)$ \\
\hline $\mathrm{C} 6-\mathrm{N} 6-\mathrm{H} 1 \mathrm{~N} 6$ & $124(3)$ & $\mathrm{C} 3-\mathrm{C} 2-\mathrm{C} 1$ & $120.0(4)$ \\
\hline $\mathrm{C} 6-\mathrm{N} 6-\mathrm{H} 2 \mathrm{~N} 6$ & $121(4)$ & $\mathrm{C} 3-\mathrm{C} 2-\mathrm{H} 2$ & $125(3)$ \\
\hline $\mathrm{H} 1 \mathrm{~N} 6-\mathrm{N} 6-\mathrm{H} 2 \mathrm{~N} 6$ & $114(4)$ & $\mathrm{C} 1-\mathrm{C} 2-\mathrm{H} 2$ & $115(3)$ \\
\hline $\mathrm{C} 5-\mathrm{N} 3-\mathrm{H} 1 \mathrm{~N} 3$ & $122(3)$ & $\mathrm{C} 2-\mathrm{C} 3-\mathrm{C} 4$ & $121.4(4)$ \\
\hline $\mathrm{C} 5-\mathrm{N} 3-\mathrm{H} 2 \mathrm{~N} 3$ & $123(4)$ & $\mathrm{C} 2-\mathrm{C} 3-\mathrm{H} 3$ & $122(3)$ \\
\hline $\mathrm{H} 1 \mathrm{~N} 3-\mathrm{N} 3-\mathrm{H} 2 \mathrm{~N} 3$ & $114(5)$ & $\mathrm{C} 4-\mathrm{C} 3-\mathrm{H} 3$ & $117(3)$ \\
\hline $\mathrm{N} 6-\mathrm{C} 6-\mathrm{N} 5$ & $118.1(3)$ & $\mathrm{C} 3-\mathrm{C} 4-\mathrm{C} 5$ & $119.7(4)$ \\
\hline $\mathrm{N} 6-\mathrm{C} 6-\mathrm{C} 7$ & $123.7(4)$ & $\mathrm{C} 3-\mathrm{C} 4-\mathrm{H} 4$ & $127(2)$ \\
\hline $\mathrm{N} 5-\mathrm{C} 6-\mathrm{C} 7$ & $118.1(3)$ & $\mathrm{C} 5-\mathrm{C} 4-\mathrm{H} 4$ & $114(3)$ \\
\hline
\end{tabular}

Hydrogen-bond geometry $\left(\AA,{ }^{\circ}\right)$

\begin{tabular}{lllll}
\hline$D-\mathrm{H} \cdots A$ & $D-\mathrm{H}$ & $\mathrm{H} \cdots A$ & $D \cdots A$ & $D-\mathrm{H} \cdots A$ \\
\hline $\mathrm{N} 1-\mathrm{H} 2 N 1 \cdots \mathrm{Cl} 2$ & $0.84(5)$ & $2.69(5)$ & $3.432(4)$ & $147(4)$ \\
$\mathrm{N} 4-\mathrm{H} 2 N 4 \cdots \mathrm{Cl} 2^{\mathrm{i}}$ & $0.84(5)$ & $2.65(5)$ & $3.465(5)$ & $162(4)$ \\
$\mathrm{N} 6-\mathrm{H} 1 N 6 \cdots \mathrm{Cl} 4$ & $0.85(5)$ & $2.58(5)$ & $3.406(4)$ & $165(4)$ \\
$\mathrm{N} 2-\mathrm{H} 1 N 2 \cdots \mathrm{Cl} 2$ & $0.82(4)$ & $2.44(4)$ & $3.240(3)$ & $168(4)$ \\
$\mathrm{N} 5-\mathrm{H} 1 N 5 \cdots \mathrm{Cl} 1$ & $0.82(5)$ & $2.43(5)$ & $3.191(3)$ & $156(4)$ \\
$\mathrm{N} 3-\mathrm{H} 1 N 3 \cdots \mathrm{Cl} 4^{\mathrm{ii}}$ & $0.88(4)$ & $2.53(5)$ & $3.390(5)$ & $166(4)$ \\
$\mathrm{N} 4-\mathrm{H} 1 N 4 \cdots \mathrm{Cl} 1$ & $0.79(4)$ & $2.79(4)$ & $3.481(5)$ & $147(4)$ \\
$\mathrm{N} 6-\mathrm{H} 2 N 6 \cdots \mathrm{Cl} 3^{\mathrm{iii}}$ & $0.82(5)$ & $2.75(5)$ & $3.516(4)$ & $156(4)$ \\
$\mathrm{N} 3-\mathrm{H} 2 N 3 \cdots \mathrm{Cl} 3$ & $0.83(6)$ & $2.61(6)$ & $3.420(5)$ & $166(6)$ \\
$\mathrm{N} 1-\mathrm{H} 1 N 1 \cdots \mathrm{Cl} 1^{\text {iv }}$ & $0.94(5)$ & $2.69(5)$ & $3.326(4)$ & $126(4)$ \\
$\mathrm{C} 7-\mathrm{H} 7 \cdots \mathrm{Cl}{ }^{3 i i}$ & $0.91(4)$ & $2.89(4)$ & $3.669(4)$ & $145(3)$ \\
\hline
\end{tabular}

Symmetry codes: (i) $-x+3 / 2, y+1 / 2,-z+3 / 2$; (ii) $-x+1 / 2, y+1 / 2,-z+3 / 2$; (iii) $-x+1,-y+1,-z+2$; (iv) $-x+1,-y+1,-z+1$. 